\title{
The Evolution of Magnetism in Iron from the Atom to the Bulk
}

\author{
Murilo L. Tiago ${ }^{(a)}$, Yunkai Zhou ${ }^{(b) *}$, M.M.G. Alemany ${ }^{(c)}$, Yousef $\operatorname{Saad}^{(d)}$, and James R. Chelikowsky ${ }^{(a, e)}$ \\ (a) Center for Computational Materials, Institute for Computational \\ Engineering and Sciences, University of Texas, Austin, TX 78712, USA. \\ (b) Department of Mathematics, Southern Methodist University, Dallas TX 75275, USA. \\ (c) Departamento de Fúsica de la Materia Condensada, Facultad de Física, \\ Universidad de Santiago de Compostela, E-15782 Santiago de Compostela, Spain \\ (d) Department of Computer Science \& Engineering, \\ University of Minnesota, Minneapolis, MN 55455, USA. \\ (e) Departments of Physics and Chemical Engineering, University of Texas, Austin, TX 78712, USA.
}

(Dated: October 18, 2018)

\begin{abstract}
The evolution of the magnetic moment in iron clusters containing 20 to 400 atoms is investigated using first-principles numerical calculations based on density-functional theory and real-space pseudopotentials. Three families of clusters are studied, characterized by the arrangement of atoms: icosahedral, body-centered cubic centered on an atom site, and body-centered cubic centered on the bridge between two neighboring atoms. We find an overall decrease of magnetic moment as the clusters grow in size towards the bulk limit. Clusters with faceted surfaces are predicted to have magnetic moment lower than other clusters with similar size. As a result, the magnetic moment is observed to decrease as function of size in a non-monotonic manner, which explains measurements performed at low temperatures.
\end{abstract}

The existence of spontaneous magnetization in metallic systems is an intriguing problem because of the extensive technological applications of magnetic phenomena and an incomplete theory of its fundamental mechanisms. Clusters of metallic atoms are important in this respect as they serve as a bridge between the atomic limit and the bulk, and can form a basis for understanding the emergence of magnetization as function of size. Several phenomena such as ferromagnetism, metallic behavior, and ferroelectricity have been intensely explored in bulk metals, but the way they manifest themselves in clusters is an open topic of debate. At the atomic level, ferromagnetism is associated with partially filled $3 d$ orbitals. In solids, ferromagnetism may be understood in terms of the itinerant electron model [1], which assumes partial delocalization of the $3 d$ orbitals. In clusters of iron atoms, delocalization is weaker owing to the presence of a surface, whose shape affects the magnetic properties of the cluster. Because of their small size, iron clusters containing a few tens to hundreds of atoms are superparamagnetic: the entire cluster serves as a single magnetic domain, with no internal grain boundaries 22. Consequently, these clusters have strong magnetic moments, but exhibit no hysteresis.

The magnetic moment of nano-sized clusters has been measured as function of temperature and size [3, 4, 5], and several aspects of the experiment have not been fully clarified, despite the intense work on the subject [6, 7, 8, 9, 10, 11]. One intriguing experimental observation is that the specific heat of such clusters is lower than

\footnotetext{
${ }^{*}$ Work done while at the Department of Computer Science \& Engineering, University of Minnesota, Minneapolis, MN 55455, USA.
}

the Dulong-Petit value, which may be due to a magnetic phase transition [4]. In addition, the magnetic moment per atom does not decay monotonically as function of the number of atoms and for fixed temperature. Possible explanations for this behavior are: structural phase transitions, strong dependence of magnetization with the shape of the cluster, or coupling with vibrational modes [4]. One difficulty is that the structure of such clusters is not well known. First-principles and model calculations have shown that clusters with up to 10 or 20 atoms assume a variety of exotic shapes in their lowest-energy configuration [12, 13]. For larger clusters, there is evidence for a stable body-centered cubic (BCC) structure, which is identical to ferromagnetic bulk iron [6].

The evolution of magnetic moment as function of cluster size has attracted considerable attention $3,4,4,5,6$, 7, 8, 9, 10, 11, 12, 13]. A key question to be resolved is: What drives the suppression of magnetic moment as clusters grow in size? In the iron atom, the permanent magnetic moment arises from exchange splitting: the $3 d_{\uparrow}$ orbitals (majority spin) are lower in energy and completely occupied with 5 electrons, while the $3 d_{\downarrow}$ orbitals (minority spin) are partially occupied with one electron, resulting in a magnetic moment of $4 \mu_{B}, \mu_{B}$ being the Bohr magneton. When atoms are assembled in a crystal, atomic orbitals hybridize and form energy bands: $4 s$ orbitals create a wide band which remains partially filled, in contrast with the completely filled $4 s$ orbital in the atom; while the $3 d_{\downarrow}$ and $3 d_{\uparrow}$ orbitals create narrower bands. Orbital hybridization together with the different bandwidths of the various $3 d$ and $4 s$ bands result in weaker magnetization, equivalent to $2.2 \mu_{B}$ /atom in bulk iron.

In atomic clusters, orbital hybridization is not as strong because atoms on the surface of the cluster have 
(a)
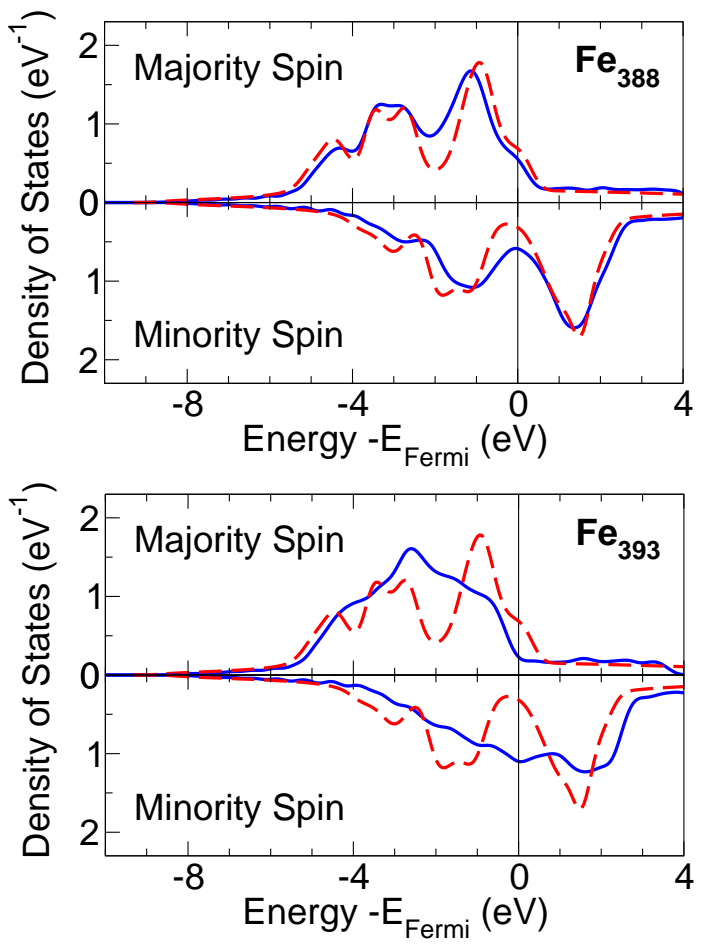

FIG. 1: Density of states in the clusters $\mathrm{Fe}_{388}$ (a) and $\mathrm{Fe}_{393}$ (b), majority spin (upper panel) and minority spin(lower panel). $\mathrm{Fe}_{388}$ corresponds to a fragment of the BCC crystal. Fe 393 has local icosahedral coordination. For reference, the density of states in bulk iron is shown in dashed lines. The Fermi energy is chosen as energy reference.

fewer neighbors. The strength of hybridization can be quantified by the effective coordination number. A theoretical analysis of magnetization in clusters and thin slabs indicates that the dependence of the magnetic moment with the effective coordination number is approximately linear [8, 10, 11]. But the suppression of magnetic moment from orbital hybridization is not isotropic 14]. For instance, if we consider a layer of atoms on the $x-y$ plane, the $3 d$ orbitals oriented on the plane (the ones with angular dependence $x y$ and $x^{2}-y^{2}$ ) will hybridize more effectively than orbitals oriented normal to the plane (with angular dependence $3 z^{2}-r^{2}$ ), because of increased overlap among orbitals on the $x-y$ plane. If the layer has infinite extent, orbitals oriented on that plane will tend to form an energy band wider than the one formed by orbitals normal to it. With a wider band, the difference in electron population between the majority spin channel and the minority spin channel is reduced, leading to reduced spin polarization and overall weaker magnetization. Reduced orbital hybridization has been observed to enhance the magnetic moment of metallic clusters [15] and thin layers [16]. As a consequence of this anisotropy, clusters with faceted surfaces are expected to have magnetic properties different from clusters with irregular surfaces, even if they have the same effective co- ordination number. This effect is likely responsible for a non-monotonic suppression of magnetic moment as function of cluster size. In order to analyze the role of surface faceting, we have performed first-principles calculations of the magnetic moment of iron clusters with various geometries and with sizes ranging from 20 to 400 atoms.

We determine the electronic structure of clusters within the framework of pseudoptentials 17 constructed using density-functional theory (DFT) 18, 19, 20]. DFT is an established theory for first-principles studies of weakly and moderately correlated electronic systems. The exchange-correlation functional used in this work employs the generalized gradient approximation (GGA) [21]. We have observed that the GGA predicts magnetic moments enhanced with respect to the simpler localdensity approximation by $2 \%$ to $10 \%$. For a fixed geometry of the cluster, we solve self-consistently the KohnSham equation on a regular grid in real space 22, 23]. Proper boundary conditions are obeyed by imposing the electronic wave functions to vanish on the boundary of a large spherical domain, which contains the system of interest. No explicit basis set is used. Numerical convergence is controlled with two parameters: the radius of the domain (typically $5 \AA$ larger than the cluster radius) and the nearest-neighbor spacing in the regular grid. For iron atoms, we use a spacing of 0.3 a.u., (approximately $0.16 \AA$ ).

We use the PARSEC code 22, 23]. This code makes use of symmetry properties of the system and very efficient techniques for solving the Kohn-Sham equation 24, 25]. Until recently, a significant fraction of numerical effort was spent in performing exact diagonalization of the Kohn-Sham equation [20, 22, 23, 26]. Curently, this step is replaced with a series of subspace filtering iterations with Chebyshev polynomials, which reduce the overall numerical effort by one order of magnitude or more 24]. This dramatic advance in methodology allows us to study confined systems with hundreds, if not thousands of atoms in a straightforward and computationally efficient manner.

Clusters of both icosahedral and BCC symmetry are explored in our work. In order to investigate the role of surface faceting, we construct clusters with faceted and non-faceted surfaces. Faceted clusters are constructed by adding successive atomic layers around a nucleation point. Small faceted icosahedral clusters exist with sizes 13, 55, 147, and 309. Faceted BCC clusters are constructed with BCC local coordination and, differently from icosahedral ones, they do not need to be centered on an atom site. We consider two families of cubic clusters: atom centered or bridge centered, respectively for clusters with nucleation point at an atom site or on the bridge between two neighboring atoms. The lattice parameter is equal to the bulk value, $2.87 \AA$. Non-faceted clusters are built by adding shells of atoms around a nucleation point so that their distance to the nucleation 


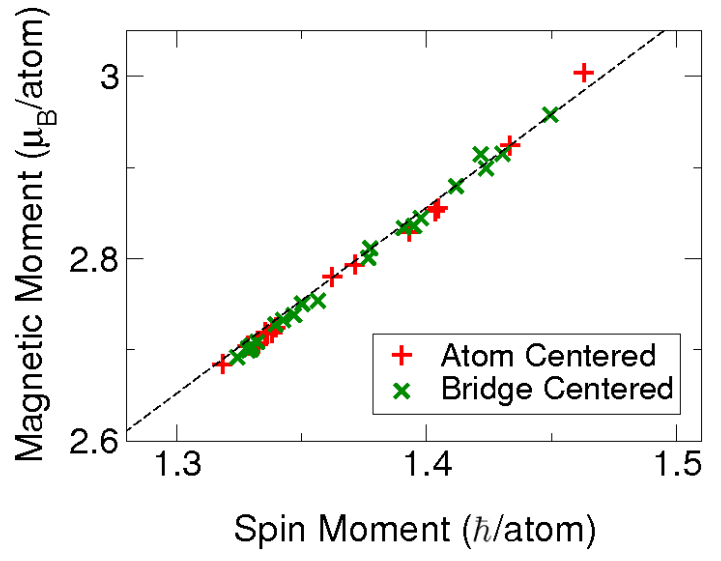

FIG. 2: Magnetic moment versus spin moment calculated for the atom-centered BCC ("plus" signs) and bridge-centered BCC (crosses) iron clusters. The approximate ratio is $M /\left\langle S_{z}\right\rangle=g_{\text {eff }}=2.04 \mu_{B} / \hbar$.

point is less than a specified value. As a result, nonfaceted clusters usually have narrow steps over otherwise planar surfaces and the overall shape is almost spherical. By construction, non-faceted clusters have well-defined point-group symmetries: $I_{h}$ or $T_{h}$ for the icosahedral family; $O_{h}$ for the atom-centered family; and $D_{4 h}$ for the bridge-centered family. Clusters constructed in that manner show low tension on the surface, making surface reconstruction less likely. Our calculations indicate that atoms on the surface feel forces weak in magnitude and directed towards the center of the cluster. Owing to the small surface/volume ratio in large clusters, the impact of surface reconstruction on those clusters will be small, if not negligible.

As clusters grow in size, their properties approach the properties of bulk iron. Figure 1(a) shows the density of states (DOS) for $\mathrm{Fe}_{388}$, with local BCC coordination. At this size range, the density of states assumes a shape typical of bulk iron, with a three-fold partition of the $3 d$ bands. In addition, the cohesive energy of this cluster is only $77 \mathrm{meV}$ lower than in bulk. This evidence suggests that interesting size effects will be predominantly observed in clusters smaller than $\mathrm{Fe}_{388}$. Figure 1(b) shows the DOS for $\mathrm{Fe}_{393}$, which belongs to the icosahedral family. This cluster has a very smooth DOS, with not much structure compared to $\mathrm{Fe}_{388}$ and bulk BCC iron. This is due to the icosahedral-like arrangement of atoms in $\mathrm{Fe}_{393}$. The overall dispersion of the $3 d$ peak ( $4 \mathrm{eV}$ for $3 d_{\uparrow}$ and $6 \mathrm{eV}$ for $3 d_{\downarrow}$ ) is nevertheless similar in all the calculated DOS.

The magnetic moment is calculated as the expectation value of the total angular momentum:

$$
M=\frac{\mu_{B}}{\hbar}\left[g_{s}\left\langle S_{z}\right\rangle+\left\langle L_{z}\right\rangle\right]=\mu_{B}\left[\frac{g_{s}}{2}\left(n_{\uparrow}-n_{\downarrow}\right)+\frac{1}{\hbar}\left\langle L_{z}\right\rangle\right]
$$

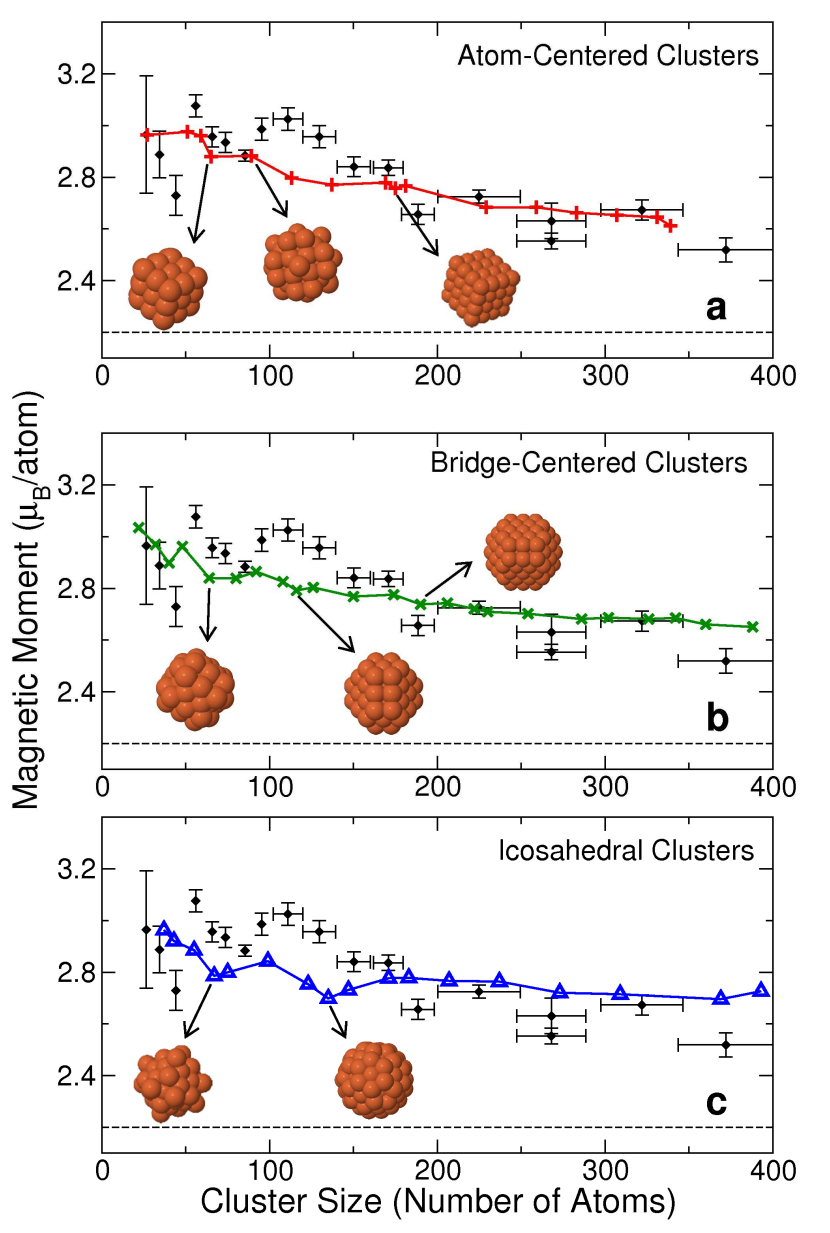

FIG. 3: Calculated magnetic moments for clusters in the atom-centered ("plus" signs, a), bridge-centered (crosses, b), and icosahedral (triangles, c) families. Experimental data [3] is shown in black diamonds with error bars. Some of the faceted and non-faceted clusters are depicted next to their corresponding data points. The dashed lines indicate the value of magnetic moment per atom in bulk iron.

where $g_{s}=2$ is the electron gyromagnetic ratio. Figure2 2 illustrates the approximately linear dependence between the magnetic moment and spin moment, $\left\langle S_{z}\right\rangle$, throughout the whole size range. This results in an effective gyromagnetic ratio $g_{\text {eff }}=2.04 \mu_{B} / \hbar$, which is somewhat smaller than the gyromagnetic ratio in bulk BCC iron, $2.09 \mu_{B} / \hbar$. The difference in ratios is probably due to an underestimation in the orbital contribution, $\left\langle L_{z}\right\rangle$. In the absence of an external magnetic field, orbital magnetization arises from the spin-orbit interaction, which is included in the theory as a model potential,

$$
V_{s o}=-\xi \mathbf{L} \cdot \mathbf{S}
$$

where $\xi=80 \mathrm{meV} / \hbar^{2}[$ ] $]$.

Figure 3 shows the magnetic moment of several clusters belonging to the three families studied: atom-centered 
BCC (top panel), bridge-centered BCC (middle panel) and icosahedral (bottom panel). Experimental data obtained by Billas and collaborators [3] is also shown. The suppression of magnetic moment as function of size is readily observed. Also, clusters with faceted surfaces are predicted to have magnetic moments lower than other clusters with similar sizes. This is attributed to more effective hybridization of $d$ orbitals along the plane of the facets. We also notice that the correlation between magnetic moment and surface smoothness is not always well defined. For instance, the cluster $\mathrm{Fe}_{175}$ in Figure 3(a) has wide facets but its magnetic moment is not much weaker than clusters with similar sizes. In Figure 31(c), clusters with strongly suppressed magnetic moment have 67 and 135 atoms, whereas clusters with wide facets and similar sizes have 55 and 147 atoms.

The measured non-monotonic behavior of magnetic moment can be attributed to the shape of the surface. Under this assumption, islands of low magnetic moment (observed at sizes 45, 85 and 188) are associated to clusters with faceted surfaces. In the icosahedral family, the islands of low magnetic moment are located around faceted clusters containing 55, 147, and 309 atoms. The first island is displaced by 10 units from the measured location. For the atom-centered and bridge-centered families, we found islands at $(65,175)$ and $(92,173)$ respectively, as indicated in Figure [3 (a,b). The first two islands are also close to the measured islands at 85 and 188 . Clearly, there is no exact superposition in the location of calculated islands and measured islands. The magnetic moment was measured in clusters at $120 \mathrm{~K}$ 3, 5]. At that temperature, vibrational modes or the occurrence of metastable configurations can shift the islands of low magnetic moment or make them more diffuse. Assuming that the non-monotonic decay of magnetic moment is dictated by the cluster shape, we also conclude that clusters with local structures different from the ones we discuss here (such as cobalt clusters with hexagonal-close packed coordination, or nickel clusters with face-centered cubic coordination) should have islands of low magnetic moment located at different "magic numbers", according to the local atomic coordination.

In summary, we discussed the behavior of magnetization in iron clusters containing 20 to 400 atoms in the light of first-principles density-functional theory. The magnetic moment is found to decay as function of cluster size in a non-monotonic fashion: clusters with faceted surfaces are predicted to have magnetic moments lower than clusters with non-faceted surfaces. As a consequence, clusters with many steps, or atoms protruding from the surface, are expected to have strong magnetic properties at low temperatures. In addition, large clusters with icosahedral structure are expected to have magnetic moments lower than clusters with BCC structure.

This work was supported by the National Science
Foundation under DMR-0130395 and DMR-0551195 and by the U.S. Department of Energy under DE-FG0289ER45391 and DE-FG02-03ER15491. Calculations were performed at the Minnesota Supercomputing Institute (MSI), at the National Energy Research Scientific Computing Center (NERSC), and at the Texas Advanced Computing Center (TACC). M.M.G.A. acknowledges support from the Spanish Ministry of Education and Science (Program "Ramón y Cajal").

[1] D. C. Mattis, The Theory of Magnetism (SpringerVerlag, Berlin, 1988), 2nd ed.

[2] C. P. Bean and J. D. Livingston, J. Appl. Phys. 30, 120s (1959).

[3] I. M. L. Billas et al., Phys. Rev. Lett. 71, 4067 (1993).

[4] D. Gerion et al., Phys. Rev. B 62, 7491 (2000).

[5] I. M. L. Billas, A. Châtelain, and W. A. de Heer, Science 265, 1682 (1994).

[6] J. A. Franco, A. Vega, and F. Aguilera-Granja, Phys. Rev. B 60, 434 (1999).

[7] A. V. Postnikov and P. Entel, Phase Transitions 77, 149 (2004).

[8] O. Šipr, M. Košuth, and H. Ebert, Phys. Rev. B 70, 174423 (2004).

[9] A. V. Postnikov, P. Entel, and J. M. Soler, Eur. Phys. J. D 25, 261 (2003).

[10] R. Félix-Medina, J. Dorantes-Dávila, and G. M. Pastor, Phys. Rev. B 67, 094430 (2003).

[11] K. W. Edmonds et al., Phys. Rev. B 60, 472 (1999).

[12] G. M. Pastor, J. Dorantes-Dávila, and K. H. Bennemann, Phys. Rev. B 40, 7642 (1989).

[13] O. Dieguez et al., Phys. Rev. B 63, 205407 (2001).

[14] P. Bruno, in Magnetismus von Festkörpern und grenzflächen, edited by P. Dederichs, P. Grünberg, and W. Zinn (IFF-Ferienkurs, Forschungszentrum Jülich, 1993), pp. 24.1-24.27.

[15] J. Bansmann et al., Surf. Sci. Rep. 56, 189 (2005).

[16] A. J. Freeman and R.-Q. Wu, J. Magn. Magn. Mat. 100, 497 (1991).

[17] N. Troullier and J. L. Martins, Phys. Rev. B 43, 1993 (1991).

[18] R. M. Martin, Electronic structure : basic theory and practical methods (Cambridge University Press, Cambridge, UK, 2004).

[19] R. G. Parr and W. Yang, Density-functional theory of atoms and molecules (Oxford Univ. Press, Oxford, UK, 1989).

[20] M. C. Payne et al., Rev. Mod. Phys. 64, 1045 (1992).

[21] J. P. Perdew, K. Burke, and M. Ernzerhof, Phys. Rev. Lett. 77, 3865 (1996).

[22] J. R. Chelikowsky, N. Troullier, and Y. Saad, Phys. Rev. Lett. $\quad \mathbf{7 2}, \quad 1240 \quad$ (1994), see http://www.ices.utexas.edu/parsec/

[23] J. R. Chelikowsky et al., Phys. Rev. B 50, 11355 (1994).

[24] Y. Zhou et al., J. Comput. Phys., in press (2006).

[25] Y. Zhou et al., Phys. Rev. E, submitted (2006).

[26] T. L. Beck, Rev. Mod. Phys. 72, 1041 (2000). 